\title{
CIFUENTES HONRUBIA, J. L. (2010): CLASES \\ SEMÁNTICAS Y CONSTRUCCIONES SINTÁCTICAS: ALTERNANCIAS LOCALES EN ESPAÑOL. LUGO, AXAC, 243 PÁGS.
}

\author{
Ruth María Lavale Ortiz \\ Universidad de Alicante \\ Ruth.Lavale@ua.es
}

La nueva obra del profesor Cifuentes es, como el mismo autor confiesa en el apartado de "preliminares", el resultado de su particular interés por el estudio de los verbos denominales locales, verbos a los que lleva unos años dedicando su trabajo gracias a un proyecto de investigación financiado por el Ministerio de Ciencia e Innovación. En esta obra el autor da cuenta de las alternancias locales que se producen en español, y muchos de los verbos denominales locales son verbos que alternan. La adaptación de unos artículos publicados años atrás configura algunos de los apartados clave de este libro que se erige como obra básica para aquellos estudios que demuestran la relación entre léxico, sintaxis y semántica.

El libro se estructura en cuatro grandes capítulos, dedicados al estudio de las clases verbales y las alternancias locales. En el primero, sobre "clases verbales y alternancias", el autor establece el marco teórico y define los conceptos básicos de alternancia verbal, realización argumental o clase verbal. Para su clasificación provisional de las alternancias verbales, José Luis Cifuentes parte de la realizada por Levin en 1993, la primera gran clasificación verbal referida al inglés. Tras señalar que quedan fuera de su clasificación aquellas alternancias en las que el verbo cambia su significado básico o las que vienen motivadas por problemas de ambigüedad, se presenta un conjunto de 59 alternancias en las que se incluyen algunas más genéricas y otras centradas en determinadas funciones sintácticas. Estas alternancias pueden estar motivadas por principios pragmáticos, discursivos, cambios aspectuales, por diferentes formas de conceptualizar una misma escena, por cambios en los pape- 
les semánticos, que dan lugar a cambios en la sintaxis, por razones sintácticas, por tipo de construcción, etc. A lo largo de esta clasificación, José Luis Cifuentes va demostrando las diferencias que su tipología posee con la realizada por Levin para el inglés y, del mismo modo, agrupa algunos tipos de alternancias que Levin muestra por separado. A continuación, el autor comenta el enfrentamiento que existe entre los modelos derivacionales y los modelos construccionales a la hora de entender y explicar las alternancias locales. Así, dentro de los modelos derivacionales el autor repasa tres perspectivas: para la lexicista, los verbos locales que alternan son verbos de cambio de localización con un componente que señala también la manera y poseen dos estructuras léxicas conceptuales distintas, pero relacionadas, ya que una está incorporada en la otra; según la perspectiva léxico-sintáctica, los verbos alternantes locales se caracterizan por tener dos construcciones con la misma representación léxica, pero cada una lexicaliza un elemento (el continente en la construcción local y el contenido en la construcción con con); finalmente, para la perspectiva sintáctica serán las propiedades aspectuales o la representación sintáctica de la estructura del evento las que permitan la alternancia local. Por lo tanto, para la propuesta derivacional, una de las estructuras, la variante local, es la básica, mientras que la otra, la variante con con, deriva de la anterior. Por su parte, los modelos construccionales afirman que las alternancias locativas tienen lugar porque un único significado verbal puede fusionarse con dos construcciones sintácticas distintas, por lo que, al contrario que con los modelos derivacionales, no hay que derivar una variante de la otra. Los modelos lexicista y construccional confluyen a la hora de otorgar importancia a los elementos del significado, puesto que son los que determinan la relación de los argumentos, pero mientras que para los lexicistas son los elementos del significado de los verbos los que indican su estructura argumental, para los construccionales es la suma de los elementos significativos de los verbos y de las construcciones sintácticas. Por último, en este primer capítulo, el profesor Cifuentes realiza la tipología de verbos con alternancias locales y, para ello, expone previamente las clasificaciones realizadas para el inglés por autores como Pinker, quien distingue seis subclases de verbos con alternancia local, y Levin, que realiza una primera clasificación en cinco subtipos aunque reconoce que hay otros grupos de verbos con alternancias locativas. La propuesta de clasificación del profesor Cifuentes distingue dos grandes grupos de alternancias motivadas por su comportamiento sintáctico: las propiamente locales, que poseen una variante en la que el sintagma viene introducido por la preposición con (a veces de), y otro grupo que alter- 
na un complemento de lugar con otro tipo de sintagma. En cada uno de estos grupos se incluyen diferentes alternancias que el autor explica detalladamente en el tercer y cuarto capítulo.

Antes de explicar detalladamente cada uno de los tipos de alternancias locales en español, el profesor Cifuentes dedica el capítulo dos de su libro a señalar cuáles son los "aspectos determinantes de las alternancias". Es aquí donde se analizan los aspectos básicos que se han tenido en cuenta para explicar las alternancias: el holismo, el cambio de estado, la noción de tema incremental, la telicidad y afección, el perfil y la perspectiva gestáltica, la consideración de una variante alternante primaria y otra derivada y las nociones de manera y resultado. Los términos de efecto holístico, cambio de estado, tema incremental y afección y telicidad hacen referencia, en última instancia, a una misma realidad: se trata de diferenciar aquellos casos en los que el conjunto de algo se ve afectado totalmente (casos en los que el argumento base actúa como complemento directo), frente a otros en los que sólo se ve afectada una parte del conjunto (casos en los que el argumento base se realiza como complemento preposicional); sin embargo, no es la realización de un argumento base como complemento directo o como preposicional lo que determina esta interpretación holística o partitiva, sino las propiedades semánticas del lexema, nuestro conocimiento del mundo o el contexto de aparición. Por su parte, a través de la perspectiva gestáltica es posible explicar la alternancia porque las dos construcciones son enfoques alternativos según se esté destacando la figura o lo que queda como fondo; algo parecido plantea la noción de perfil, ya que lo que varía con las alternancias es la parte de la cadena causal que se perfila con el evento. A continuación, se aborda una de las cuestiones esenciales que se han tratado a la hora de explicar las alternancias: el hecho de si una de las construcciones es primaria y la otra derivada. Tras repasar las consideraciones de ciertos autores sobre esta cuestión y teniendo en cuenta la información que a este respecto puede aportar la historia de la lengua, la conclusión a la que se llega es que no hay una variante que prime sobre la otra. Finalmente, se aborda cómo las nociones de manera y resultado han servido para explicar las alternancias: según autoras como Levin y Rappaport, para que un verbo alterne debe tener un componente manera (manera de causación o desplazamiento); sin embargo, el profesor Cifuentes afirma que algunos verbos que alternan tienen el rasgo manera, otros tienen el rasgo resultado y también hay verbos que alternan y no suponen ningún cambio de estado, por lo que la solución más lógica es buscar la explicación en cada uno de los subtipos de alternancia que él propone; de esta forma se observa la 
importancia de los rasgos manera y resultado, pero dentro de cada tipo particular de verbos alternantes.

En el tercer capítulo se aborda el primer tipo de alternancias locales: las que poseen una variante en la que el sintagma está introducido por con. Para ello, se parte de la relación entre conceptos como posesión, existencia y localización. Así, se afirma que la localización es cognitivamente anterior a la posesión y a la existencia, porque si podemos localizar algo es porque ese algo existe y si poseemos algo es porque lo poseído está localizado en el poseedor. Desde el punto de vista conceptual, por tanto, las construcciones locativas, posesivas y existenciales están mutuamente relacionadas y muestran un comportamiento regular en ciertos usos. Asimismo, se muestran las diferencias entre localización y atribución: la localización es la relación existente entre entidades y lugares y la atribución es la relación entre entidades y propiedades. Con estos aspectos teóricos claramente definidos, el profesor Cifuentes explica los diferentes tipos de alternancias locales. El primer subtipo, el de las alternancias predicativas (en las que se dedican unas páginas a las cuestiones de qué elementos pueden funcionar como predicativos, a la distinción entre complemento predicativo y complemento de modo y a qué variantes con de las alternancias locales podemos considerar predicativos), evidencia la relación que existe entre los conceptos de posesión, existencia y localización: en estos verbos, se expresa localización y posesión y se atribuye una propiedad a un objeto por dicha localización y posesión. Se trata de verbos en los que una variante indica predicación locativa (Tiene granos en la cara) y otra señala la atribución de esa propiedad local al elemento poseedor (Tiene la cara con granos); este tipo de alternancias no había sido puesto de manifiesto con anterioridad y, mientras que la variante local puede darse en lenguas como el inglés, alemán, árabe, polaco, ruso, árabe y el conjunto de las lenguas románicas, entre otras, la estructura predicativa preposicional solamente es posible en las lenguas románicas. El segundo tipo de alternancias locales es el de las ablativas, en las que se produce la remoción de un elemento locatum de una localización; así, en ejemplos como Limpió el polvo de la mesa y Limpió la mesa de polvo observamos que la localización puede expresarse como objeto directo o como complemento oblicuo. En este tipo de alternancia, la relación de posesión-localización es ya existente, puesto que es el punto de partida de la acción, y debe existir en el significado de estos verbos un componente manera y la posibilidad de un determinado resultado para que puedan alternar. El tercer tipo de alternancias locales, las de objeto construido, se caracteriza porque la localización-posesión de los elementos que funcionan como contenedor- 
contenido es el objeto de la acción verbal. En este subgrupo, la construcción del objeto en un determinado lugar puede hacerse física (de forma natural, como en sembrar, o artificial, como en edificar) o mentalmente (idear); también se incluyen aquí los verbos de impresión de imagen, como grabar, cercanos a los de construcción mental, y otros del tipo rociar, en los que la figura se extiende sobre la base que contiene el objeto localizado. En cuarto lugar se encuentran las alternancias de verbos denominales locatum (engrasar), que lexicalizan el elemento contenido; con estos verbos son posibles alternancias del tipo Embaldosó el salón con gres frente a Embaldosó gres en el salón. Sin embargo, los verbos denominales de localización (embarcar) que lexicalizan el elemento continente no parecen posibilitar la alternancia local, salvo contadas excepciones que el autor comenta. En quinto lugar se explican las alternancias de fusión de verbo con relacionante, en las que el verbo expresa el hecho del desplazamiento o localización y la relación entre figura y base; estas alternancias, que se organizan en seis subtipos, pueden tener una alternancia local como las vistas hasta el momento (sobreimprimir) $\mathrm{u}$ otros tipos de alternancia (recíproca en enfrentar o partitiva en abordar, encabezar). Por último, el profesor Cifuentes aborda las alternancias que tienen un esquema de imagen "en derredor", en las que no hay un elemento dentro de otro, sino alrededor de otro, de manera que el elemento que está alrededor tiende a integrar a la base que incluye (Ceñir el talle con su brazo); en este caso, la diferencia en las construcciones reside en la manera de ser de los objetos.

Una vez vistas las alternancias locales más habituales, el último capítulo se centra en las alternancias locales que alternan un complemento de lugar con otro tipo de sintagma, pero que no poseen variación de un complemento introducido por con. El primer gran grupo lo conforman las alternancias partitivas, donde encontramos, además de las construcciones de acusativo partitivo, las construcciones partitivas direccionales y las de desplazamiento violento. Las construcciones de acusativo partitivo dan lugar a alternancias del tipo Le mordió la oreja y Le mordió en la oreja, en las que tenemos una estructura directa en la que el objeto se ve completamente afectado por la acción y una construcción con locativo que posee una interpretación partitiva que afecta a un lugar. Por su parte, las construcciones partitivas direccionales son menos productivas que las anteriores, pero análogas a ellas: Apuntó (la pistola) a la cara de su enemigo y Apuntó a su enemigo en la cara (con la pistola); la diferencia reside en que en las anteriores el objeto afectado venía dado como objeto directo y en éstas es un complemento de lugar a dónde. En este primer grupo se incluyen también las alternancias generadas por verbos 
que expresan un desplazamiento violento, como Lo arrojó a la cara de su enemigo y Se lo arrojó a su enemigo en la cara, que, a diferencia de las construcciones partitivas vistas, no poseen una figura implicada en la acción, sino que está explícita en forma de objeto directo. Las alternancias partitivas tienen en común las relaciones de posesión inalienable con una parte del cuerpo, por lo tanto, un esquema de localización-atribución. El segundo grupo de estas alternancias locales sin variante con lo conforman las alternancias posesivas; se trata de construcciones en las que aparece un sintagma nominal que indica una propiedad de un sujeto y, en la construcción alternante, se separan sujeto y característica. Aquí, el autor diferencia tres subtipos de alternancias según si el elemento poseedor y lo poseído aparecen como objeto directo (Admiro en Cristina su bondad e inocencia, Admiro la bondad e inocencia de Cristina y Admiro a Cristina por su bondad e inocencia), como sujeto de un verbo transitivo (Las locuras de Elisa asustan a Manuel, Elisa asusta a Manuel con sus locuras y Sus locuras asustan a Manuel) o como sujeto de un verbo intransitivo (El precio de los sellos bajó, Los sellos bajaron de precio y Su precio bajó). Observamos aquí, de nuevo, la relación entre posesión y localización. Un tercer grupo de estas alternancias es el de las alternancias recíprocas, en las que ya no están presentes las relaciones local-posesivas del esquema contenedor/contenido. Se trata de verbos que poseen un significado local y cuya alternancia se debe a la reversibilidad o reciprocidad: Añadir sal al caldo y Añadir caldo a la sal, esto es, la alternancia se debe a la posibilidad de focalizar un elemento u otro como figura o base de la localización. Finalmente, se abordan otras alternancias que, a diferencia de las vistas en este capítulo y en el anterior, se dan con verbos que no tienen tres argumentos y en las que no aparece un sintagma con, aunque sigue estando implicado un complemento de lugar; se trata de las alternancias locales de sujeto, otras alternancias de objeto directo, alternancias de localización temporal y alternancias intransitivas. En estas últimas alternancias, los procesos metafóricos y metonímicos son claves para la articulación de la construcción sintáctico-semántica.

El libro se cierra con un epílogo, en el que el autor resume los contenidos más relevantes tratados a lo largo de los cuatro capítulos, y el apartado de referencias bibliográficas, sin duda de gran valor para todos los interesados en la materia.

En definitiva, en la nueva obra que José Luis Cifuentes nos presenta quedan explicadas las alternancias locales más habituales y las menos frecuentes, las clases verbales que dan lugar a estas alternancias y los rasgos que permiten dichas alternancias. El mundo que rodea el fenómeno de las alternancias 
es complejo, puesto que no se ciñe únicamente a la sintaxis: estudiar y analizar las alternancias, tal y como el profesor Cifuentes lo ha hecho, implica observar el léxico, la semántica, la sintaxis y cuestiones pragmáticas. No podemos entender las alternancias sin abordar temas como el de estructura argumental o clase verbal y nociones como localización, posesión, atribución y existencialidad. La forma sencilla y didáctica con la que el autor explica este fenómeno, revisando otros tantos aspectos y partes del lenguaje y de la lengua, ha dado lugar a una obra de obligada referencia en los estudios actuales sobre léxico, sintaxis y semántica. 